\title{
WIND SPEED VECTOR RESTORATION ALGORITHM
}

\author{
Nikolay Baranov ${ }^{1}$, Gleb Petrov ${ }^{2}$, Ilia Shiriaev ${ }^{2}$ \\ ${ }^{1}$ Dorodnicyn Computing Centre, FRC CSC RAS \\ 2 JSC "International Aeronavigation Systems Concern" (IANS)
}

\begin{abstract}
Impulse wind lidar (IWL) signal processing software developed by JSC «BANS» recovers full wind speed vector by radial projections and provides wind parameters information up to $2 \mathrm{~km}$ distance. Increasing accuracy and speed of wind parameters calculation signal processing technics have been studied in this research. Measurements results of IWL and continuous scanning lidar were compared. Also, IWL data processing modeling results have been analyzed.
\end{abstract}

\section{INTRODUCTION}

An effective means for determining the wind profile of the surface layer of the atmosphere is a Doppler wind lidar. Currently, the most widely spread lidars are operating with infrared laser beam at a wavelength of 1.54 microns. The lidar beam is scattered by aerosol particles which are present in the atmosphere.

VAD and DBS are the atmosphere scanning modes by the lidar laser beam. They provide aggregated data reception on the values of the radial projection of the wind speed in the measurement direction. This data is further converted into the value of the full vector of wind speed for all heights of scanning [1].

In a continuous circular scan the wind speed at a fixed height of its radial projection on the beam will be described by a harmonic law [1]:

$$
\begin{gathered}
y=-u \cdot \sin \theta \cdot \cos \varphi- \\
-v \cdot \cos \theta \cdot \cos \varphi-w \cdot \sin \varphi,
\end{gathered}
$$

where $y$ - lidar measured value of the radial projection of the wind speed, $u, v, w$ - full vector of wind speed components, $\theta$ azimuth of the measurement direction, $\varphi$ - deviation angle from the vertical measurement direction.

The major problem with wind speed and direction remote measurement, is that the measurements are at the same height have spatiotemporal distribution. For example, when measuring the wind speed at a height of 1000 meters with the value of the deflection angle from the vertical of 20 degrees, the distance between the points of measurement can reach $730 \mathrm{~m}$. In the case of spatially inhomogeneous wind field large spatial distribution of measuring points can generate additional error when dealing with the recovery of the wind velocity vector problem. Therefore, the measurement model used for the restoration of the wind velocity vector, must take into account the spatial inhomogeneity of the wind field.

\section{METHODOLOGY}

In this paper, the wind field model is based on the hypothesis of uncorrelated linear variable for each horizontal coordinate of the wind speed horizontal component variability. The wind speed vertical component is assumed spatially uniform throughout the volume of measurement at each height. The wind speed gradients for the directions can be set.

Let us set:

- $u_{0}, v_{0}$ - wind speed horizontal component spatially averaged over the measurement volume at a fixed height values of the

- $u_{x}$ - gradient alteration of the $u$-wind speed component along the axis $\mathrm{x}$,

- $u_{y}$ - gradient alteration of the $u$ - wind speed component along the axis $\mathrm{y}$,

- $v_{x}$ - gradient alteration of the $v$ - wind speed component along the axis $\mathrm{x}$,

- $v_{y}$ - gradient alteration of the $v$ - wind speed component along the axis $y$.

The usage of a gradient wind field model allows to obtain additional wind characteristics that determine horizontal wind shear at each height.

The linear model of the wind speed gradients in the directions of the horizontal plane provides for the total value of the wind speed component along each axis represents an additive mixture of the half value of the speed and gradient. For example, 
the value of wind speed along the $\mathrm{x}$-axis components can be written as:

$$
\left\{\begin{array}{l}
u(x)=u_{0}+x \cdot u_{x} \\
v(x)=v_{0}+x \cdot v_{x}
\end{array}, x \in(-R . . R),\right.
$$

where $R$ - the radius of the scan circle at a fixed height.

Linear coordinates are functions of the azimuthal measurements direction angle. It provides a linear system of equations for determining the unknown parameters of the wind field, but the system is inconsistent. If you write the equations (1) for the speed radial projection, taking into account the impact of linear gradients of wind speed in the directions in the horizontal plane, you can get:

$$
\begin{aligned}
& y=-\left(u_{0}+u_{x} \cdot R \cdot \sin (\theta)+u_{y} \cdot R \cdot \cos (\theta)\right) \\
& \cdot \sin \theta \cdot \cos \varphi- \\
& -\left(v_{0}+v_{x} \cdot R \cdot \sin (\theta)+v_{y} \cdot R \cdot \cos (\theta)\right) \\
& \cdot \cos \theta \cdot \cos \varphi-w \cdot \sin \varphi
\end{aligned}
$$

It is obvious, that after the brackets removal, the coefficients of $u_{x}$ and $v_{x}$ are the equal, which makes it impossible for an independent analysis of wind speed gradients in the directions.

Let us analyze the possibility of the harmonic approximation of the dependence of the lined coordinates of a bell shaped function which simulates the scan beam movement in the azimuthal angle.

Consider the possibility of function bell shaped usage in the following equations:

$$
\left\{\begin{array}{c}
f_{x}(\theta)=R \cdot \exp \left(-\beta \cdot \theta^{2}\right) \\
f_{y}(\theta)=R \cdot \exp \left(-\beta \cdot\left(\theta-\frac{\pi}{2}\right)^{2}\right)
\end{array}\right.
$$

where $\beta$ - the normalizing index that determines the beam width, $R$ - the radius of the scan circle at a fixed height..

The values of the azimuthal angle range from $-\pi$ to $\pi$ radians. The normalization index $\beta$ must provide a single value of the $f_{x}$ function in 0 radians point and $f_{y}$ function when the azimuth angle of $\pi / 2$ radians. In addition, the values of bell shaped functions should fall to 0 within the range of the azimuth angle change. When $\beta$ is close to the 1 , the listed conditions are satisfied.
The wind speed components at an arbitrary point, determined by an azimuth angle $\theta$ taking into account (3) can be written as

$\left\{\begin{array}{l}u=u_{0}+u_{x} R \exp \left(-\theta^{2}\right)+u_{y} \operatorname{Rexp}\left(-\left(\theta-\frac{\pi}{2}\right)^{2}\right) \\ v=v_{0}+v_{x} \operatorname{Rexp}\left(-\left(\theta-\frac{\pi}{2}\right)^{2}\right)+v_{y} R \exp \left(-\theta^{2}\right)\end{array}\right.$

The equation for the wind speed radial projection in the scanning direction taking into account the uniformity of the vertical component of the wind speed is the following:

$$
y=-\left(u_{0}+u_{x} R \exp \left(-\theta^{2}\right)+u_{y} R \exp \left(-\left(\theta-\frac{\pi}{2}\right)^{2}\right)\right) \text {. }
$$

$\cdot \sin \theta \cos \varphi-$

$$
-\left(v_{0}+v_{x} \operatorname{Rexp}\left(-\left(\theta-\frac{\pi}{2}\right)^{2}\right)+v_{y} \operatorname{Rexp}\left(-\theta^{2}\right)\right) \text {. }
$$

$\cdot \cos \theta \cos \varphi-w \cdot \sin \varphi$

For the exact restoration of the seven parameters of the numerical simulation model it is not enough to have a connection of the radial projection from the azimuthal angle. Solving the wind field parameters restoration problem by an available set of the wind speed radial projections, the solutions of the optimization problem is linearly independent on $\left(\mathrm{u}_{0}, \mathrm{v}_{0}\right)$ and $\left(\mathrm{u}_{\mathrm{x}}, \mathrm{u}_{\mathrm{y}}, \mathrm{v}_{\mathrm{x}}, \mathrm{v}_{\mathrm{y}}\right)$ parameters. The model, includes an additional optimization parameter - $\mathrm{w}$, which does not have any function of the azimuth angle. Thus, the determination of the wind speed vertical component in the recovery issue, taking into account the spatial inhomogeneity is possible only with the introduction of additional modulation for the vertical component of the wind speed. This modulation is possible by changing the scan beam

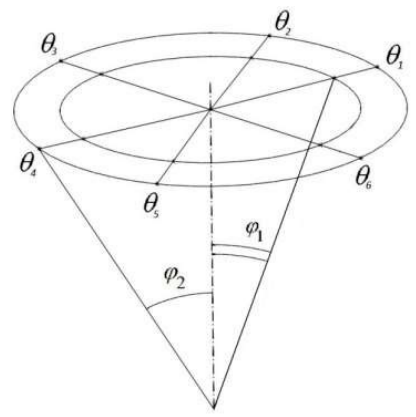

angle of elevation.

Figure 1 Lidar scan pattern 
The scan algorithm should foresee the possibility of working with variable angles of elevation. The scan pattern for two fixed values of the angle of elevation and six values of azimuth are shown in the figure 1 .

The equation (3) can be shown the following way:

$$
\begin{aligned}
& y=u_{0} x_{i j 1}+u_{x} x_{i j 2}+u_{y} x_{i j 3}+v_{0} x_{i j 4}+ \\
& v_{x} x_{i j 5}+v_{y} x_{i j 6}+w x_{j 7} \\
& \text { where }\left\{\begin{array}{c}
x_{i j 1}=-\sin \theta_{i} \cdot \cos \varphi_{j} \\
x_{i j 2}=-\exp \left(-\theta^{2}\right) \cdot \sin \theta_{i} \cdot \cos \varphi_{j} \\
x_{i j 3}=-\exp \left(-\left(\theta-\frac{\pi}{2}\right)^{2}\right) \cdot \sin \theta_{i} \cdot \cos \varphi_{j} \\
x_{i j 4}=-\cos \theta_{i} \cdot \cos \varphi_{j} \\
x_{i j 5}=-\exp \left(-\left(\theta-\frac{\pi}{2}\right)^{2}\right) \cdot \cos \theta_{i} \cdot \cos \varphi_{j} \\
x_{i j 6}=-\exp \left(-\theta^{2}\right) \cdot \cos \theta_{i} \cdot \cos \varphi_{j} \\
x_{j 7}=-\sin \varphi_{j}
\end{array}\right.
\end{aligned}
$$

With the known values of the angle of azimuth and elevation angle, the coefficients $\mathrm{x}_{1 . .7}$ are permanent. The problem is reduced to finding the vector component of the wind speed values $\left(\mathrm{u}_{0}, \mathrm{v}_{0}, \mathrm{w}\right)$ and indicators of the wind field inhomogeneity $\left(\mathrm{u}_{\mathrm{x}}, \mathrm{u}_{\mathrm{y}}, \mathrm{v}_{\mathrm{x}}, \mathrm{v}_{\mathrm{y}}\right)$, satisfying the equation (5) with the available measurement results. The result of measurements is the set of values of the radial projection of the wind speed $y$, obtained from the corresponding azimuth angle values and elevation, thus $x_{1.7}$. The values of the radial speed projections are attached to the same range of marks along the beam, thus the same height of scan.

For an exact solution of the equation with seven unknowns seven measurements of the radial projection of the wind speed at different values of the angle of azimuth and at least two elevation angles are necessary. This solution will be precise from a mathematical point of view, but is subject to distortion in view of the real atmospheric turbulence, as well as the presence of technical measurement system noise and interferences.

\section{RESULTS}

Taking into account the physical and technical aspects of the noise impact, redundant inputs are used in the experiments. In other words, the measurement is carried out over seven measurements of the wind speed radial projection. This system of equations becomes redundant and allows the plurality of solutions. To select the optimal solution the object function is introduced.

The object function is to reflect the discrepancy of the resulting function to actually measured data in the experiment. For solving this problem it is required to find such a combination of $\mathrm{u}, \mathrm{u}_{\mathrm{x}}, \mathrm{u}_{\mathrm{y}}, \mathrm{v}, \mathrm{v}_{\mathrm{x}}, \mathrm{v}_{\mathrm{y}}, \mathrm{w}$ that minimizes error recovery. Since all the equations are linear, then the search for suitable solutions optimal linear regression algorithm is based on the method of least squares.

According to the obtained values of the wind speed component mean horizontal wind speed, vertical wind speed and wind direction in the horizontal plane can be calculated:

- horizontal wind speed: $H=\sqrt{u^{2}+v^{2}}$;

- vertical wind speed: $V=w$;

- wind direction: $D=\arccos \left(\frac{v}{V}\right)$.

Furthermore, during the problem solution 4 spatial non-uniformity of the wind field are determined the gradients of the wind speed in the horizontal plane.

From the perspective the most effective algorithm developing for space scanning, it seems the optimal to do two turns on the azimuth angle with a fixed position of the elevation. The design of an impulse wind lidar includes an xy-scanner, and such scanning algorithm can be implemented on the currently existing equipment.

Developed new model were tested on real lidar data, which were obtained during atmosphere scanning with two different elevations: 50 and 70 degrees. Wind speed vector restoration algorithm was applied to this data for three and seven parameter models with fitting function (1) and (4) respectively. Examples of results are shown in the figure 2 .

Seven parameters model (red curve) improves outlier detection, prevents false filtering and better fits experimental data that decreases wind parameters restoration error, while three parameters model (gray curve) approximates data less accurately.

Statistical information for each model is shown in the table 1 . 

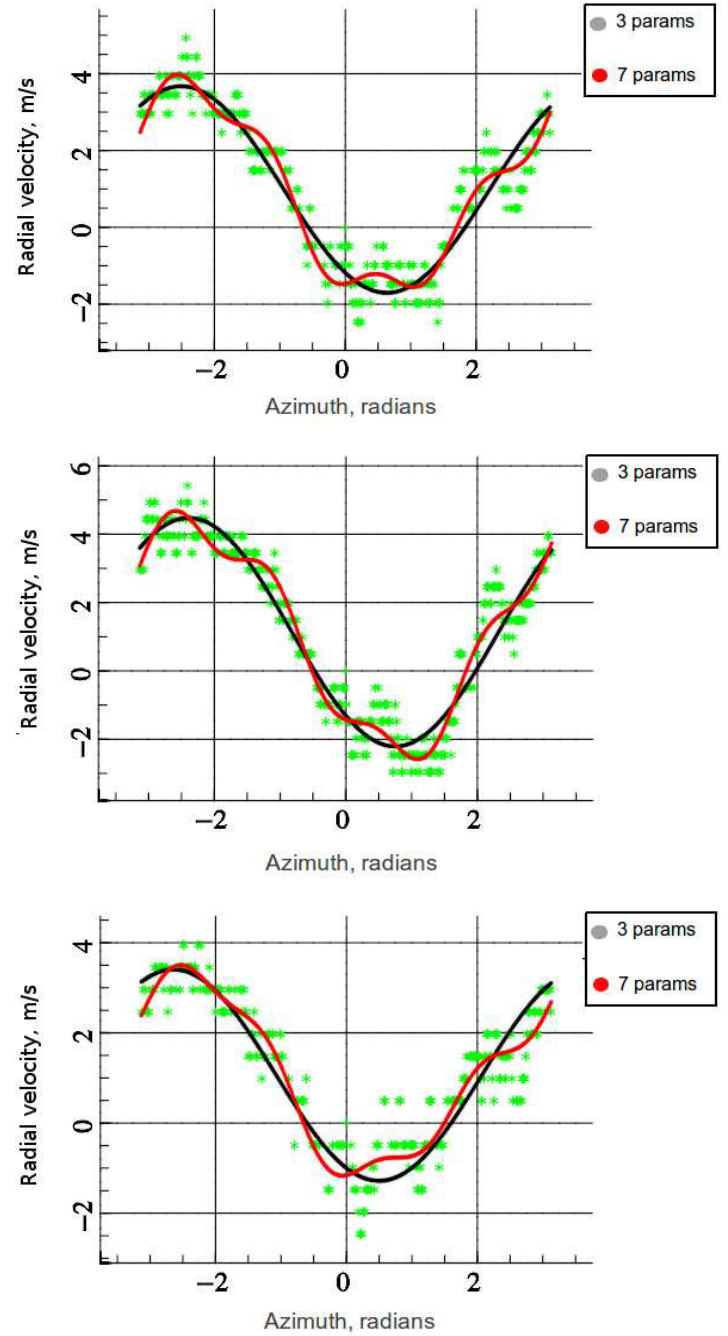

Figure 2 Comparison of three and seven parameter models

Table 1

Statistics for three and seven parameters models

\begin{tabular}{|c|c|c|}
\hline Statistics & $\begin{array}{c}\text { Seven } \\
\text { parameters } \\
\text { model }\end{array}$ & $\begin{array}{c}\text { Three } \\
\text { parameters } \\
\text { model }\end{array}$ \\
\hline $\mathrm{R}^{2}$ adjusted & 0,95 & 0,83 \\
\hline Average error & 0,39 & 0,62 \\
\hline Standard error & 0,48 & 0,74 \\
\hline
\end{tabular}

New model lets us:

- refine description of processes occurring in the atmosphere;
- acquire additional information about wind spatial distribution.

\section{CONCLUSIONS}

From the mathematical modeling, and taking into account the impact of noise, it implies that the value of the elevation angle should be 20 and 40 degrees from the vertical direction, and the azimuthal direction should be evenly spaced in a circle scan. The number of fixed positions azimuth angle is 5-7. Such scanning order will ensure the scan data processing, taking into account the spatial distribution of the wind field, and in addition, will not lead to a decrease in the wind speed and direction results calculation updating frequency on the output of the algorithm.

\section{ACKNOWLEDGEMENTS}

The work was partially supported by the Russian Foundation for Basic Research, project 16-07-01072.

\section{References}

[1] Christian Werner, Dr. Claus Weitkamp, 2005: Lidar. Range-Resolved Optical Remote Sensing of the Atmosphere, Springer New York, pp 325-354.

[2] ICAO Doc 9817 - Manual on Low-Level Wind Shear.

[3] Willett H. C., Sanders F. Descriptive Meteorology, Academic Press Inc. New York. 1959.

[4] Boilley A., Mahfouf J.-F. Wind shear over the Nice C`ote d'Azur airport: case studies, Nat. Hazards Earth Syst. Sci., 13, 22232238, 2013.

[5] Barthelmie R.J. The effects of atmospheric stability on coastal wind climates, Meteorol. Appl. 6, 39-47. 1999. 